Artículo original

\title{
CONTROL DE PRESIONES Y REDUCCIÓN DE PÉRDIDAS EN EL SISTEMA DE DISTRIBUCIÓN DE AGUA POTABLE DEL SECTOR VI - AUGUSTO B. LEGUÍA DE TACNA
}

CONTROL OF PRESSURES AND REDUCTION OF LOSSES IN THE DRINKING WATER DISTRIBUTION SYSTEM IN SECTOR VI - AUGUSTO B. LEGUÍA DE TACNA

\author{
Cesar Arturo Avalos Bravo ${ }^{1}$ \\ (D) https://orcid.org/ 0000-0003-4411-7529 \\ William Albert Flores Landa ${ }^{2}$ \\ (1) https://orcid.org/ 0000-0002-4041-3307 \\ Jimmi Yury Silva Charaja ${ }^{3}$ \\ (1) https://orcid.org/ 0000-0001-5973-7670
}

Información del artículo: Recibido: 21/01/2021 Aceptado: 01/06/2021 Publicado: 28/06/2021

\author{
1,2 Escuela de Ingeniería Civil, Universidad Privada de Tacna \\ ${ }^{3}$ Docente en la Escuela de Ingeniería Civil, Universidad Privada de Tacna \\ E-mail: ${ }^{1}$ arturo.ab23@ hotmail.com, ${ }^{2}$ a7berth@gmail.com, ${ }^{3}$ jsch84@hotmail.com
}




\section{Resumen}

Se estudió el control de pérdidas y presiones de agua potable, por medio de la identificación del índice de pérdidas a nivel de sector urbano, evaluación de la funcionabilidad de las válvulas reductoras de presión y del sistema de distribución de agua potable, del sector VI de la ciudad de Tacna, con el fin de mejorar la eficiencia operativa y Mitigar las pérdidas en las redes de Agua Potable. El estudio aplicó una metodología de tipo descriptivo, de diseño de campo (cuantitativo y cualitativo), empleando la observación y el cálculo matemático de resultados, cuyo instrumento fue el material de medición. La investigación concluye que, para poder tener una mayor eficiencia operativa es necesario primero medir el punto de partida en cuanto se refiere al índice de pérdidas de agua potable, y como consecuencia de este establecer un plan de trabajo para mitigar estas pérdidas, ya sea por medio de la evaluación hidráulica, regulación de presiones u otra actividad operativa.

Palabras claves: índice de pérdidas, Modelación hidráulica, presión estática, presión dinámica, válvula reductora de presión, caudal promedio, macro medición, agua no facturada.

\section{Abstract}

The control of drinking water losses and pressures was studied by identifying the loss index at the urban sector level, evaluating the functionality of the pressure reducing valves and the drinking water distribution system in sector VI of the city of Tacna, with the aim of improving operational efficiency and mitigating losses in the drinking water networks. The study applied a descriptive methodology, field design (quantitative and qualitative), using observation and mathematical calculation of results, whose instrument was the measurement material. The research concludes that, in order to have greater operational efficiency, it is first necessary to measure the starting point in terms of the rate of drinking water losses, and as a result establish a work plan to mitigate these losses, either by means of hydraulic evaluation, pressure regulation or other operational activity.

Key Words: loss index, hydraulic modeling, static pressure, dynamic pressure, pressure reducing valve, average flow, macro measurement, unbilled water. 


\section{Introducción}

La presencia de pérdidas de agua en sistemas de abastecimiento urbano es un problema de eficiencia operacional, que se debe estudiar de forma integral ya que comprende múltiples facetas. Para las empresas prestadoras del servicio de agua potable. Por ello es de vital importancia identificar las pérdidas que ocurren en sus procesos de captación, conducción, potabilización y distribución de agua a todos sus usuarios.

La EPS TACNA S.A, cuenta con 07 sectores operacionales conteniendo cada uno 5 sub sectores, encontrando como crítico al subsector 20 sector $\mathrm{VI}$, evidenciando deficiencias en el servicio de distribución de agua potable, como sobre presiones y discontinuidad de servicio, provocando una serie de reclamos por parte de los usuarios.

Para ello se plantea el control de pérdidas y presiones, identificando el indicador de perdidas como primer paso, posteriormente realizando un modelo hidráulico del sub sector 20 sector $\mathrm{VI}$, con la finalidad de evaluar la funcionabilidad de las válvulas reductoras de presión existentes, y por medio de esto reducir de manera óptima las pérdidas de agua en la red de distribución del subsector operacional mencionado, y finalmente con este modelo hidráulico evaluar la eficiencia de la operación del sistema en condiciones típicas.

Con esto se plantea el "Control de presiones y reducción de pérdidas en el sistema de distribución de agua potable del Sector VI - Pueblo Joven Augusto B. Leguía en la Ciudadde Tacna".

La ciudad de Tacna situada en la zona costera del Perú, es abastecida por 02 fuentesde agua superficial y 02 fuentes subterráneas, Esta agua cruda es procesada, convertida a agua potable y distribuida por la Entidad Prestadora de Servicios de Saneamiento (Tacna EPS TACNA S.A). Esta cuenta con una red de distribución mixta, dividida en siete (07) sectores operacionales. Identificando dentro de estos, el subsector 20 sector VI, este sub sector cuenta con 31,864 km de Tuberiasde agua potable, siendo estas $18,634 \mathrm{Km}$ de asbesto de cemento (AC) y 13,230 Km de policloruro de vinilo (PVC) representando un $64 \%$ tuberías de AC y $36 \%$ tuberías de PVC, a lo largo del tiempo (entre los años 2015 al 2019) este sector ha ido presentandodeficiencias en el abastecimiento del servicio de agua potable, siendo estas, la bajacontinuidad de servicio y la diversidad de presiones detectadas en la zona (de 8 m.c.a a 38 m.c.a),

\section{Figura 1}

Esquema de sector Fuente propia

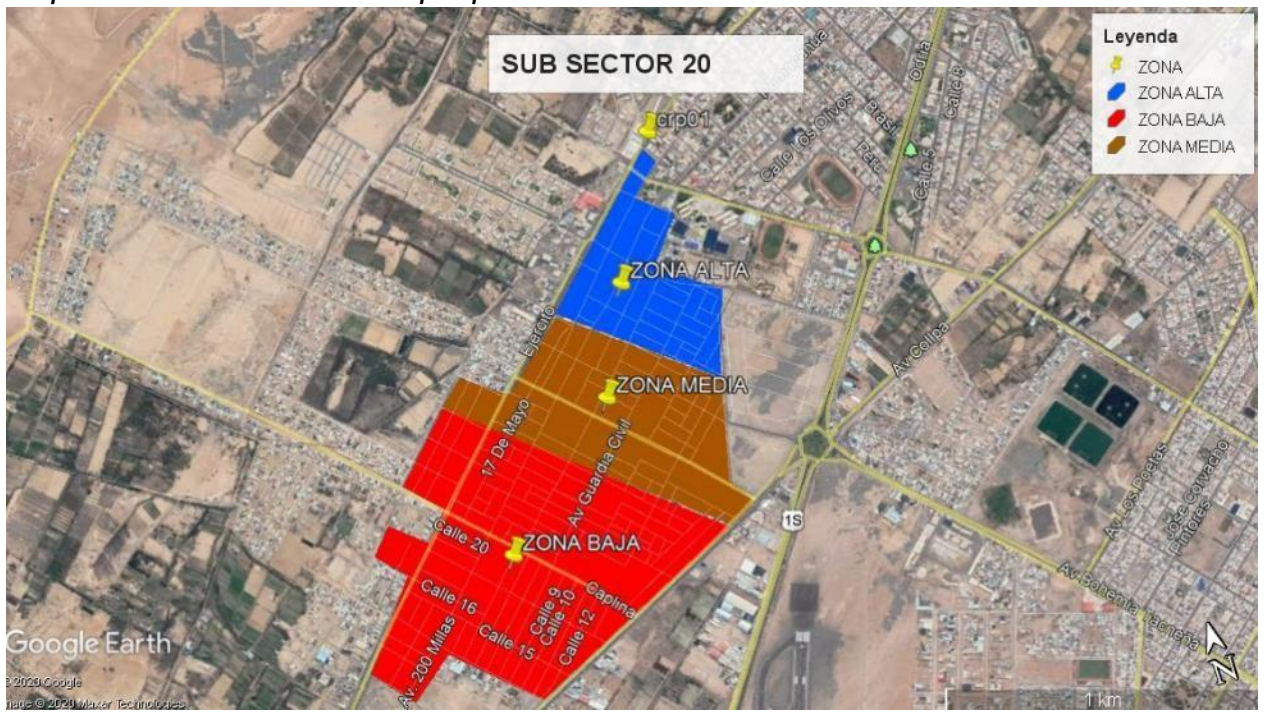


Para conocer más sobre la situación en la que se encuentra la zona de investigaciónSe recompilo data de la EPS TACNA. S.A. durante El periodo 2015-2018, podemos observar que aparentemente estas fugas han reducido, favorablemente para la empresa encargada del suministro, sin embargo la caída abrupta en el reporte de las fugas,sin haberse ejecutado programa alguno de detección de fugas ni renovación de redes, llevaa suponer que el sistema es óptimo, al no haber reportes de fugas de agua, sin embargo debido a la discontinuidad del servicio y las quejas por presiones bajas en ciertas zonas delsub sector 20 sector $\mathrm{VI}$, despierta la duda sobre la presencia de fugas llevando a la pregunta

¿Cuál es el índice de pérdidas de agua potable en el sector?, ya que si el sistema no presenta fugas de agua, ¿porque no se cumple con la demanda de la población en el sub sector?

Un análisis del promedio de conexiones por año durante elperiodo 2015-2019.correspondiente al subsector 20 sector IV. Este análisis muestra que existe un incremento de 150 conexiones en promedio del periodo 2015-2019, sin embargo, se puede observar que las conexiones del año 2019 se han reducido notablemente en comparación con los años 2017 y 2018.

Así mismo analizando la continuidad de servicio durante el periodo 2015-2019, se observa. Un deceso de horas de servicio de un pico máximo de 24 horas a 12 horas. Esto causa extrañeza el número promedio de conexiones del año 2016 es de 3,338 y cuenta con una continuidad de 24 horas, pero al 2019 con solo 1 conexión más que el año 2016, esta continuidad se ha reducido a 12 horas.

Tabla 1

Análisis de data histórica (2015-2019)

\begin{tabular}{cccccc}
\hline $\begin{array}{c}\text { Sector } \mathrm{VI} \\
\text { subsector } 20\end{array}$ & 2015 & 2016 & 2017 & 2018 & 2019 \\
\hline $\mathrm{N}^{\circ}$ de conexiones & 3265 & 3338 & 3383 & 3449 & 3339 \\
Horas de servicio & 21 & 24 & 14 & 13 & 12 \\
\hline
\end{tabular}

Resumiendo claramente el deceso de la continuidad de servicio lo cual si consideramos que el volumen entregado el año 2019 es mayor al que se dotaba el año 2015 podemos deducir que este sistema presenta pérdidas de agua, las cuales causan la disminución de la continuidad de servicio. El reservorio, se cuenta con 3 válvulas reductoras de presión cada una controlando un sistema

\section{Figura 2}

Áreas de influencia de VRP, Fuente propia

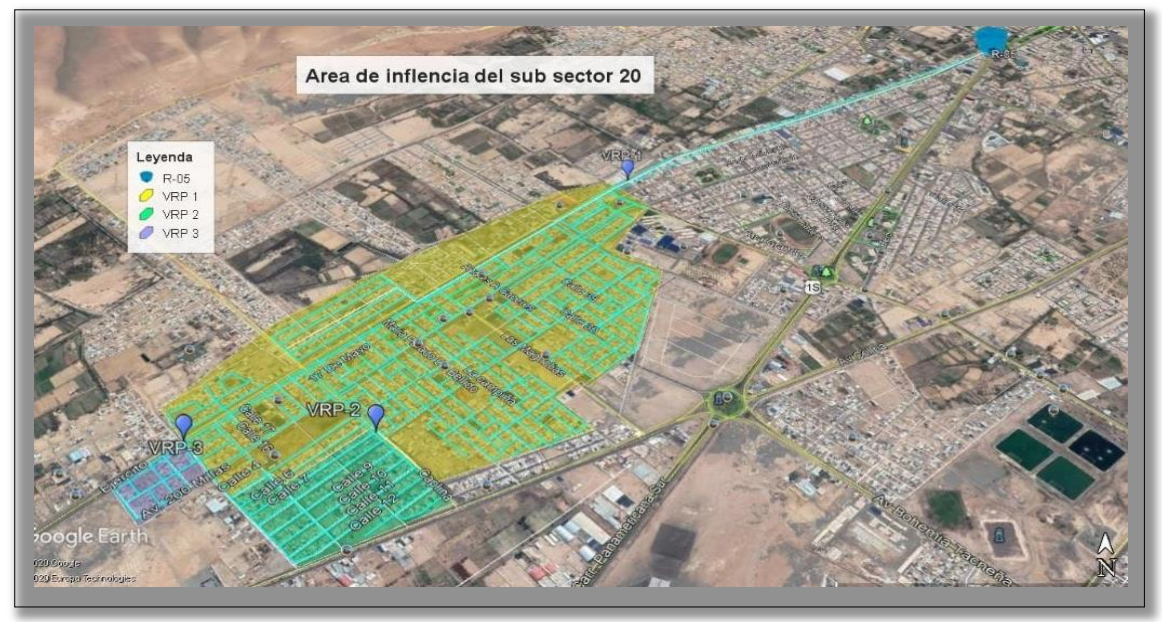

Cada área de influencia en la que impactan las válvulas reductoras de presión, estas áreas están distinguidas por colores, lográndose ver que la VRP $n^{\circ} 1$ tiene un área de influencia amplia marcada de 
color amarillo, con casi a un $70 \%$ de todo el sub sector 20 . Naciendo la pregunta ¿las válvulas reductoras de presión funcionan adecuadamente para el control de presiones en todo el sub sector 20? Así mismo al identificar la funcionabilidad de las válvulas reductoras de presión y si estas operan de manera oportuna para el control de presiones surgiría la pregunta, ¿¿El sistema de distribución existente en el subsector operacional 20 sector VI de la red de distribución de la ciudad de Tacna es el adecuado?, ¿Cuál es el efecto de las presiones y reducción de pérdidas en el sistema de distribución de agua potable del Sector VI - Pueblo Joven Augusto B. Leguía en la Ciudad de Tacna?

Es necesario conocer el efecto de las pérdidas y presiones, sobre la infraestructura hidráulica y la población del sub sector 20 sector VI, ya que la entidad EPS TACNA S.A, cuenta con información limitada. Las pérdidas y presiones de agua en el sub sector 20 sector $\mathrm{VI}$, han generado una serie de deficiencias en su infraestructura hidráulica partiendo desde las fallas en redes. Principales, acomedidas hasta las conexiones domiciliarias. Ocasionando problemas en la infraestructura vial pública y el medio ambiente. Así mismo, esto ha generado infinidad de conflictos con la población, por el mal servicio brindado, producto de estas pérdidas y presiones de agua. Esto a su vez, ocasiona que los medios económicos se gasten en acrecentar la producción de agua para compensar las pérdidas, cuando podrían invertirse en conservar oampliar la infraestructura actual.

Bartolín, (2011) ha presentado el software GISRed, cuya funcionalidad permite generar, simular y calibrar modelos de las redes desde el entorno SIG, prestando una gran ayuda a la hora de efectuar diagnósticos y elaborar planes paraotras prestaciones enfocadas a asistir al usuario en tareas más específicas propias de la gestión técnica de las redes, tales como la gestión de averías, de la demanda, del control de fugas mediante sectorización, el análisis de la modelación diaria o el consumo nocturno. Desarrolló un software GISRed, que funciona como una extensión del sistema de información geográfica ArcView, para facilitar la construcción y calibración de los modelosmatemáticos de redes de distribución. Vegas (2012), desarrolla un algoritmo potente que permita diseñar sectores hidrométricos mediante divisiones de la red de abastecimiento de agua en zonas y convertirse en una herramienta que controle loscaudales y regule las presiones, obteniendo como beneficio la detección y reparaciónde tuberías con fugas, reducir el agua que se pierde, tener una mejor planificación del sistema de la red de abastecimiento y controlar de forma activa y permanente delas pérdidas de agua a través de las fugas para mantener un rendimiento óptimo del balance hídrico del sistema; encontró es que, para sectorizar una red, es necesario desarrollar un modelo matemático que nos permita analizar el funcionamiento de la red actual y poder generar un cambio el árboldirigido de mínimo coste (longitud, diámetro, caudal, velocidad, pérdida de carga unitaria y resistencia hidráulica), con lo cual los diseños de las delimitaciones pueden variar.

Campbell (2013), realizó una investigación tuvo como resultado que la sectorización de la red de distribución de agua potable representa una opción estratégica que puede llevarse a cabo con múltiples objetivos, que van desde la mejora del Control Activo de Fugas (CAF) hasta el control de la calidad del agua, aparte de favorecer el tratamiento de algunos problemas en las mismas (fugas, calidad del agua, reparaciones, etc.) , debido a la reducción dimensional implícita enla sectorización; sin embargo, su implantación cambia su comportamiento hidráulico,dado que al implicar el cierre de válvulas en las tuberías, se rompe el principio de redundancia hidráulica que tienen las redes malladas, haciéndolas más vulnerables a entrar en escenarios de desabastecimiento ante la falla de uno o más de sus elementos. Fragoso (2015) tuvo como finalidad realizar la sectorización de la red de agua potable para mejorar la entrega, distribución y control de caudales, se utilizó el programa EPANETpara realizar sus análisis hidráulicos. La calibración del modelo se efectuó en relación con las pérdidas de agua y el caudal, considerando datos medidos del emisor, los resultados obtenidos a través de la EPANET es que se recuperó el volumen de aguapor día de $14262 \mathrm{m3}$, que corresponde a un gasto medio recuperado de $165 \mathrm{l} / \mathrm{s}$ debido a la reducción de fugas de red del 76,8 \% (sin regular presión) al 25,3\% (regulando presión). Con esto se comprobó la hipótesis inicial de que a través de la sectorización se mejora la eficiencia hidráulica, debido al control de las fugas al detectarlas de manera electrónica en tiempo real, y se tiene una mayor facilidad para repararlas. 


\section{Objetivo}

Controlar las presiones y reducción de pérdidas en el sistema de distribución de aguapotable del Sector VI - Pueblo Joven Augusto B. Leguía en la Ciudad de Tacna.

\section{Metodología}

Investigación explicativa se utilizó para expresar el comportamientode una variable (pérdidas de agua) en función de una a más variables independientes (presión, continuidad de servicio). Este tipo de estudio permitió explicar, comprender e interpretar el por qué ocurren las pérdidas de agua y en qué condiciones se dan. Con este tipo de investigación se identificaron las causas del problema, para posteriormente plantear soluciones. Los niveles de investigación alcanzados en la presente tesis son los siguientes:

a. Perceptual: este fue el nivel inicial ya que este nivel correspondió al planteamiento inicial de la problemática en la que se encontraba la zona de estudio.

b. Aprehensivo: En este nivel de investigación se realizó una investigación sobre los antecedentes de la zona de estudio en cuanto a presiones e índice de pérdidas de agua.

c. Comprensivo: Este nivel de investigación se alcanzó al monitorear los caudalesde abastecimiento al sub sector 20 sector IV.

d. Integrativo: Este nivel de investigación se alcanzó al realizar la evaluación del sistema con el modelamiento hidráulico e identificar la perdida de presión

Población y/o muestra de estudio: El sub sector 20, sector VI, este compuesto por las zonas, Pueblo Joven Augusto Leguía, Asociación de vivienda los granados, Asoc. De vivienda los CIPRESES, Asoc. De vivienda señor de locumba. Con una cantidad de 18,171.0 habitantes, los cuales representan 4732 conexiones domiciliarias de las cuales un $80 \%$ son domésticas y un $20 \%$ comerciales. La ciudad de Tacna cuenta aproximadamente con 286,240 habitantes, de los cuales el subsector 20 sector VI representa el $6 \%$ de la población total, siendo 17,174.4 habitantes. La estructura hidráulica principal del sector 20 sector VI, está compuesta por 31.864 km de tubería (18,364 Km de PVC y 13,230 Km de asbesto de cemento) con diámetros de $90 \mathrm{mma} 200 \mathrm{~mm}$. Además de tres válvulas reductoras de presión situadas estratégicamente en laszonas bajas del sub sector.

Las técnicas que se emplean son de análisis situacional y observación experimental con apoyo del personal técnico de la EPS TACNA S.A. Así como también con apoyo de equipos sofisticados para la recolección de datos en la red. Los datos obtenidos durante el proceso de la investigación se organizarán y procesarán de forma manual y digital con el propósito de presentarla información de manera ordenada, clara y sencilla.

Para el procesamiento de datos del presente estudio se hará uso de lossiguientes programas:

- Microsoft Office Excel2010: Registro de información sobre la base de los formatos empleados. Este procedimiento permitirá configurar la matriz de sistematización de datos que se adjuntaran al informe.

- $\quad$ Autocad 2018: AutoCAD es un software de diseño asistido por computadorautilizado para dibujo 2D y modelado 3D.

- Microsoft Office Word 2010: Es un programa informático orientado al procesamiento de textos. Fue creado por la empresa Microsoft

- Google Earth: Es un programa informático que muestra un globo virtual que permitevisualizar múltiple cartografía, con base en la fotografía satelital. Soporte para la extensión espacial de PostgreSQL, PostGIS. Manejo de archivos vectoriales Shapefile, Arclnfo coverages, Mapinfo, GRASS GIS, DXF, DWG, etc. Soporte para un importante número de tipos de archivos raster(GRASS 
GIS, GeoTIFF, TIFF, JPG, etc.)

- Wáter Gems V8i: Es un instrumento eficiente para poder diseñar modelos hidráulicos referentes a redes de distribución de agua potable y simular escenarios favorables y desfavorables para los sistemas de agua potable. Fue creadopor la empresa Bentley Systems, Incorporated.

Para el trabajo en campo se utilizan algunos instrumentos de medición yseñalización como los siguientes:

- Hoja de Registro de datos: Se utiliza para tener un control de la data en campo.

- Manómetro: Este instrumento sirve para medir presiones en tiempo real sus unidades de medida se dan em PSI y BAR.

- Data logger: La EPS TACNA S.A. cuenta con equipos registradores con transductores de presión (data logger), estos equipos permiten. Medir la presión en periodo extendido. Los equipos almacenan losdatos cada $1 \mathrm{~min}, 2 \mathrm{~min}, 5 \mathrm{~min}$, etc. Según la necesidad y programación del usuario. Esta medición continua permite monitorear laspresiones y las horas de servicio. En base a las presiones (presión cero sin servicio).

- Medidor de caudal portátil: Este equipo permite medir los caudales en tránsito dentro de las tuberías por medio de sensores ultrasónicos que se insertan en la superficie de las tuberías a través de estos sensores el medidor registra eltránsito de los caudales en tiempo real.

- Plano de distribución: Se utiliza para tener conocimiento e identificar la infraestructura sanitaria en campo y en gabinete.

\section{Procesamiento y análisis de datos}

Para calcular el índice de pérdidas o agua no facturada fue necesario recompilar data del subsector 20 sectores VI. La data a utilizar es: Volumen Facturado (acumulado mes) y Volumen de ingreso al sistema (acumulado mes).

Para obtener el volumen facturado es necesario recompilar la data real de facturación del área correspondiente en este caso esta información la brindo la división de catastro comercial entregando la información sobre el volumen facturado en sector Vlsubsector 20, al mes de marzo. Este cuadro muestra la cantidad de usuarios y el volumen que han facturado estos usuarios al mes de marzo el cual está representado en $\mathrm{m}^{3}$. Para obtener la información del volumen inyectado al sistema de distribución se recurrió a la Div. de distribución y control de pérdidas de la EPS TACNA. Con apoyo del especialista del área se obtuvo la información que fue descargada de un equipo macro medidor de caudal que se encuentra al ingreso del sector el cual tenía unadata acumulada del caudal entregado al sistema al mes de marzo. Este esquipo registra un caudal cada $15 \mathrm{~min}$, para lo cual es necesario hacer un ejercicio para calcular el volumen acumulado en este periodo de tiempo (15 min).

Tabla 2

Cálculo de volumen de ingreso al sistema

\begin{tabular}{ccc}
\hline Fecha/Hora & Caudal & Vol Acumulado \\
\hline $03 / 03 / 201900: 00$ & 19,44 & 17500 \\
$03 / 03 / 201900: 15$ & 22,22 & 20000 \\
$03 / 03 / 201900: 30$ & 33,33 & 30000 \\
$03 / 03 / 201900: 45$ & 51,67 & 46500 \\
$03 / 03 / 201901: 00$ & 21,67 & 19500 \\
\hline
\end{tabular}

Caudal: $19.44 \mathrm{l} / \mathrm{s}$

$19.44 \mathrm{l} / \mathrm{s} * 60 \mathrm{~s} * 15 \mathrm{~min}=17500$ litros este dato nos indica el volumen acumulado en un periodo de 15 minutos, este mismo ejercicio se repitió para todos los datos que arrojoel macro medidor. 
Tabla 3

Análisis de volumen facturado

\begin{tabular}{ccc}
\hline Rutas & Mar-19 \\
& Cantidad Usuarios & VF MAR $(\mathrm{m} 3)$ \\
\hline 8 & 496 & 5981 \\
9 & 626 & 6602 \\
10 & 595 & 8998 \\
11 & 1067 & 11176 \\
12 & 348 & 4624 \\
19 & 186 & 2361 \\
20 & 1414 & 15442 \\
Total & 4732 & 55184 \\
\hline
\end{tabular}

Este cuadro muestra la cantidad de usuarios y el volumen que han facturado estos usuarios al mes de marzo el cual está representado en $\mathrm{m}^{3}$. Evaluar el sistema de distribución del Sub sector 20, Sector VI Pueblo Joven Augusto B. Leguía en la Ciudad de Tacna mediante un modelohidráulico.

Identificar la funcionabilidad de las válvulas reductoras de presión del sub sector 20 sector VI, por medio de un modelo hidráulico en WATERGEMS V8I. Para evaluar la funcionabilidad de las válvulas reductoras de presión se plantea elaborar un modelo hidráulico existente en la plataforma WATERGEMS V8I, para ello se requiere recopilación de la data indispensable:

- Catastro actualizado y geo referenciado

- Plano de elevaciones

- Variables del sistema de distribución

- Operación del sistema

En el caso de la EPS TACNA, no se contó con un catastro compatible con la plataforma WATERGEMS V8I. Es por ello que, como primer paso se realizará la migración del catastro técnico de la EPS TACNA S.A, al software WATERGEMS con las mismas características que cuenta el sistema de distribución. Luego insertamos las curvas de nivel en el modelo para trabajar en una superficie.

Identificación de variables del sistema de distribución del sector VIsubsector 20 de la EPS Tacna

Para esto se recompila la data histórica de presiones a fin de poder analizarla y observar las variaciones a lo largo del tiempo. Luego de identificar las variables, Insertamos las demandas variables del sistema al año 2015 y año 2019, analizando el escenario más desfavorable para el sistema como el $\mathrm{QMH}$, finalmente, el diseño con la ayuda de Watercad para la simulación de las redes de agua potable. Habiendo modelado el sistema de distribución de agua potable de sector VI subsector 20 se obtiene como resultado la funcionalidad de las válvulas reductoras de presión y necesidad de reducir presiones en las zonas que se logren identificar.

Para evaluar el sistema de distribución del sub sector 20 sector VI, se simuló el sistema en los caudales QP, QMH y QMIN.

\section{Resultados}

Identificar el índice de perdidas (ANF) del sub sector 20 sector VI 
Tabla 4

Cálculo de ANF

\begin{tabular}{lclccc}
\hline \multicolumn{5}{c}{ Mar-19 } \\
\hline Q (I/s) & Horas deservicio & Vol dotado/mes $(1 / \mathrm{s}$ & Vol.dot/mes & Vol. Facturado & ANF \\
\hline 29.21 & 9 & 66388500 & 66389 & 55184 & $17 \%$ \\
\hline
\end{tabular}

Realizando el cálculo obtenemos que el índice de pérdidas o agua no facturada enel sector VI sub sector 20 es de $17 \%$. Este índice de pérdidas de $17 \%$ representa un volumen de $11,205 \mathrm{~m}^{3}$ por mes, que si lo representamos en pérdidas económicas con una tarifa promedio de consumo residencial doméstico (S/.1.233) sería S/. 13,815.77 al mes. Evaluar el sistema de distribución del Sub sector 20, Sector VI Pueblo JovenAugusto B. Leguía en la Ciudad de Tacna, 2019" mediante un modelo hidráulico.

Evaluación de funcionabilidad de válvulas reductoras de presión: Esta válvula recibe una presión de 48 m.c.a y la reduce a 13 m.c.a aguas abajo el área de influencia de esta válvula es de aproximadamente el $60 \%$ de todo el sub sector 20 . Los reportes arrojan que esta válvula controla las presiones de 12 m.c.a la mínimay la máxima 49 m.c.a. Esto quiere decir que esta válvula no logra minimizar las presiones en toda su áreade influencia. Quedando la zona de asociación de vivienda el cacique, con presiones sobre los 40 m.c.a en periodo dinámico

VRP n02: Esta válvula recibe una presión de 38 m.c.a y la reduce a 7 m.c.a aguas abajo el área de influencia de esta válvula es de aproximadamente el $30 \%$ de todo el sub sector 20 . Los reportes arrojan que esta válvula controla las presiones de 7 m.c.a la mínima yla máxima 21 m.c.a.; esto quiere decir que esta válvula logra minimizar las presiones en toda su área de influencia.

VRP n03: Esta válvula recibe una presión de 47 m.c.a y la reduce a 9 m.c.a aguas abajo el área de influencia de esta válvula es de aproximadamente el $10 \%$ de todo el sub sector 20 . Los reportes arrojan que esta válvula controla las presiones de 9 m.c.a la mínima yla máxima 16 m.c.a; esto quiere decir que esta válvula logra minimizar las presiones en toda su área de influencia.

Evaluando el modelo hidráulico se confirmó la eficiencia de las válvulas reductorasexistentes, pero a su vez se identificó que existe un sector que requiere una válvula reductora de presión. Se muestran las 03 VRP existentes y sus áreas de influencia, existen influencias controladas en las presiones del Sector VI sub sector 20 Pueblo JovenAugusto B. Leguía en la Ciudad de Tacna, 2019. Sin embargo, con el modelamiento hidráulico se pudo identificar que la VRP $n^{\circ} 1$ no logra controlar las presiones en toda su área de influencia. Teniendo un sector con presiones estáticas de 40 a 47 m.c.a, esto se presenta, debido a la gran cobertura de estaVRP, la configuración de redes y la topografía. la norma OS.050 en el Item 4.5. menciona: "La presión estática no será mayor de 50 m en cualquier punto de la red. En condiciones de demanda máxima horaria, la presióndinámica no será menor de $10 \mathrm{~m}$.

Si consideramos lo mencionado con referencia a la norma OS 050 , viendo que el rango de presiones máximas oscila entre 40 a 47 m.c.a, se puede deducir que las presiones son aceptables, sin embargo, al tener un $64 \%$ de la infraestructura hidráulica con tuberías de asbesto de cemento, con una antigüedad mayor a 20 años, Las condiciones de operación de la infraestructura actual no son las mismas, así como su resistencia. Asi mismo al tener una gran área de influencia se aprecia que la válvula reductora de presión 1 no logra controlar las presiones, por ello se plantea sub dividir el área de influencia de la VRP 1, instalando una (1) VRP y cerrando 02 válvulas de corte

La válvula reductora a propuesta es: 01 Válvula reguladora de presión (VRP) $160 \mathrm{~mm}$ H.D

Para verificar la eficiencia de la propuesta se analiza el área de influencia de la VRP $n^{\circ} 1$, con la VRP PROPUESTA con una consigna aguas debajo de 10 m.c.a, y se obtienen presiones desde 9 m.c.a a 31 m.c.a la máxima en toda el área de influencia, con esto podemos comprobar la eficiencia de la propuesta.

Evaluar el sistema de distribución del sub sector 20 sector VI, por medio del modelamiento hidráulico 
en WATERGEMS V8I, Luego de procesar el modelo hidráulico con un caudal promedio de consumo, verificamos, que actualmente el sistema requiere no es el ideal, ya que se detectó que requiere la instalación de una válvula reductora de presión, a fin de mantener controladaslas presiones en el área de influencia de la VRP $n^{\circ} 1$.

\section{Discusión}

Esta discusión está referida a los resultados obtenidos con el modelamiento hidráulico, en los que se identifican presiones estáticas elevadas de 42 m.c.a. lo cualtienen consecuencias físicas que afectan a las redes, más aun considerando la antigüedad de estas y la existencia de fugas además de identificar zonas que requieren intervenciónpara una solución óptima. Después de los resultados finales optimizados con WaterCAD se observa que el valor promedio de presiones esde 38.80 m.c.a. y que la velocidad flujo promedio es de $0.68 \mathrm{~m} / \mathrm{s}$ lo cual demuestra que todo el nuevo sistema planteado tiene la configuración tal que cumple hidráulicamente con unbuen funcionamiento.

Esta discusión realiza una comparación entre la utilización de método computacional Epanet (Fragoso 2015). A diferencia del WaterCAD, el método computacional EPANET, no presenta un algoritmo de multi-iteración es por eso que se realiza un diseñoóptimo, con una iteración manual así como refiere a Fragoso, que en su contenido recomienda el aislamiento de sectores que presentan diferencia altimétrica considerable para obtener un mejor funcionamiento, por lo quese usó esta recomendación y se obtuvo de resultados promedios en presión de nudos el valor de 40.29 m.c.a. y de velocidad de flujo el valor de $0,72 \mathrm{~m} / \mathrm{s}$. Expresiones numéricas que son una variación porcentual de $3.92 \%$ en presión con respecto a los resultados por Watercad y $11,91 \%$ con respecto a la velocidad, lo cual se establece como aceptable dentro de las consideraciones estadísticas.

A pesar de ser el método computacional de cálculo hidráulico en redes más reciente, no posee las mismas cualidades de respuesta que WaterCAD, se entiende que sigueen desarrollo; el antecedente internacional 3 presenta una metodología que coincide mucho con la interfaz y la interacciona de Watdis ya que de igual manera se plantea una sectorización aislada de las redes de tuberías, añade un hidrante para satisfacer las condiciones actuales y futuras de lapoblación; por lo que la propuesta de Watdis en su versión de diseño optimo muestra un hidrante adicional, y este resultado aproxima mucho a los valores obtenidos anteriormente. Con loque se refiere a velocidad de flujo en tuberías presenta un valorpromedio de $0,74 \mathrm{~m} / \mathrm{s}$, cumpliendo con el mínimo de $0,60 \mathrm{~m} / \mathrm{s}$ de la normativa; de igual manera como valor promedio de presión expresa 39,04 m.c.a, por lo que es una propuesta que también se puede considerar.

\section{Conclusiones}

El efecto de las pérdidas de agua en el sistema de distribución del sub sector 20 sector $\mathrm{VI}$, se manifiesta con la baja de la continuidad del servicio y la caída de presiones, así mismo el impacto de presiones dentro de un sistema de agua potable son un indicador de la eficiencia operativa. Se calculó un índice de pérdidas de $17 \%$, que muestra un nivel de pérdidas relativamente bajo, pero es necesario tomar en cuenta que este sector no cuenta con un abastecimiento de servicio de 24 horas, su continuidad de servicio es de 9 horas a nivelde sub sector y 12 horas a nivel de sector VI. Este índice de pérdidas de $17 \%$ representa un volumen de $11,205 \mathrm{~m}^{3}$ por mes, que si lo representamos en pérdidas económicas con una tarifa promedio de consumo residencial doméstico $(S / 1,233)$ sería S/ 13,815.77 al mes.

Las presiones de agua en el sub sector 20 sector VI son aceptables, pero, requieren analizarse a detalle, ya que en el modelo hidráulico se identificó que entre las 00:00:00 a 3:00:00 am se genera una presión estática de 42 m.c.a. que si tomamos en cuenta la existencia de fugas y la relación existente 
entre presión/fuga, esta estaría generando pérdidas elevadas durante ese lapso de tiempo, se concluye que el área de influencia de la válvula reductora de presión 1 es demasiado amplia para ser controlada por una sola válvula, por ello se plantea una propuesta sub dividiendo el área de influencia, de tal forma que esta pueda ser controlada con mayor eficiencia. Es necesario apoyarnos de un modelo hidráulico para poder evaluar el comportamientode un sistema hidráulico durante un periodo estático y dinámico, teniendo en cuenta siempre el criterio técnico, los resultados del comportamiento de velocidades en un periodo dinámico, cada línea representa el resultado de velocidades en una determinada hora, los picos más altos son las líneas anaranjadas (tubería con velocidades de $2.6 \mathrm{~m} / \mathrm{s}$ en el periodo de máximo consumo de5:00:00 am a 9:00:00 am; con el modelo hidráulico se pueden plantear alternativas de solución para poderoptimizar el sistema de distribución.

Así se recomienda a la entidad prestadora de servicios EPS TACNA realizar una evaluación mensual del indicador de pérdidas para así poder mantener o reducir este indicador; analizar el impacto económico de las pérdidas de agua potable, a fin depoder tener en cuenta el gasto operativo. Reducir las presiones a menos por lo menos en un rango de 15 a 30 a finde minimizar la fatiga en la infraestructura hidráulica. Se recomienda la instalación de una VRP de HD DN $160 \mathrm{~mm}$. Y actualizar el modelo hidráulico a medida que se generen modificacionesen la infraestructura hidráulica y alimentar el modelo hidráulico con data real de campo, para obtenerresultados óptimos.

\section{Referencias Bibliográficas}

(F Y H) Fluidos y Herramientas. (2018). Medidores Para Agua. Equipos de medición, http://fluidosyherramientas.com/medidores-de-agua/125/medidores-para-agua.

Bayona, J. (2018). Índice de agua no contabilizada. Perú:

Bentley. (2016). Software de análisis y diseño de sistemas de distribución de agua. Obtenidode Software de análisis y diseño de sistemas de distribución de agua: https://www.bentley.com/es/products/product-line/hydraulics-and-hydrologysoftware/watergems

DeVellis, R. (1991). Scale Development: Theory and Applications. Newberry Park: Sage. EPS Tacna S.A. (2013-2043). Plan Maestro Optimizado Actualizado 2013-2043. Tacna: EPS Tacna S.A.

Fallis, P., Hübschen, K., \& Oertlé, E. (2011). Guía para la reducción de las pérdidas de agua. republic, Frankfurt am Main: Rohland\&more, Offenbach/Alemania.

Jiménez, J. (2013). Manual para el diseño de sistemas de agua potable y alcantarilladosanitario. México: Universidad Veracruzana.

Marco Castro D., P. D.-I. (2016). Sobre La Modelación Hidráulica En Obras De Saneamiento Básico. Quito - Ecuador: Departamento De Ciencias Del Agua.

Ministerio de Servicios y Obras Públicas. (2004). Instalaciones de agua - Diseño para sistemas de agua potable. Bolivia: IBNORCA.

Ministerio de Vivienda, Construcción y Saneamiento. (2006). OS.0100 Consideraciones Básicas De Diseño De Infraestructura Sanitaria. En C. y. OS.0100

Ministerio de Vivienda, Consideraciones Básicas De Diseño De Infraestructura Sanitaria (pág. OS.0100). LIMA: Ministerio de Vivienda, Construcción y Saneamiento.

Ministerio de vivienda construcción y saneamiento. (2006). OS.050 Redes de distribución de agua para consumo humano. Lima: ministerio de vivienda construcción y saneamiento.

$\begin{array}{lllrl}\text { Narváez, } & \text { R. } & \text { (2019). Libro Abastecimiento de Agua. Ancash: Obtenido } \\ & \text { de: } & & \text { https://es.slideshare.net/freddyacunavilla/250603337- }\end{array}$ libroabastecimientodeaguaricardonarvaez. 
Patrick Fallis, Katja Hübschen, Emmanuel Oertlé,. (2011). Guía para la reducción de laspérdidas de agua. republic, Frankfurt am Main: Rohland\&more, Offenbach/Alemania. TECFLUID. (2018). medidores electromagnéticos en línea y de inserción. tecfluid the art of measuring, https://tecfluid.com/caudalimetros-electromagneticos-en-linea-o-insercion/. Terán, I. J. (2012). Manual Para El Diseño de Veracruz: desconocida.

Ulsen, M. A. (2017). "Reducción de pérdidas en sistemas. Santiago: Pontificia Universidad Católica de Chile.

Wikipedia. (23 de setiembre de 2019). Obtenido de wikipedia.: https://es.wikipedia.org/wiki/Electrov\%C3\%A1lvula 